\title{
Local Stability Analysis of an Infection-Age Mathematical Model for Tuberculosis Disease Dynamics
}

\section{${ }^{1}$ ASHEZUA, TT; ${ }^{2}$ AKINWANDE, NI; ${ }^{3}$ ABDULRAHMAN, $\mathbf{S} ;{ }^{4}$ OLAYIWOLA, RO; ${ }^{5}$ KUTA, FA}

\author{
${ }^{1}$ Department of Mathematics/Statistics/Computer Science, Federal University of Agriculture, Makurdi, Nigeria \\ ${ }^{1}$ Email: ttashezua@gmail.com \\ ${ }^{2,3,4}$ Department of Mathematics, Federal University of Technology, Minna, Nigeria \\ ${ }^{2}$ Email: aninuola@gmail.com; ${ }^{3}$ Email: siraja_enagi@yahoo.com; ${ }^{4}$ Email: olayiwolarasaq@yahoo.com.uk, \\ ${ }^{5}$ Department of Microbiology, Federal University of Technology, Minna, Nigeria \\ ${ }^{5}$ Email:kutafaruk@yahoo.com
}

\begin{abstract}
An infection age structured mathematical model for tuberculosis disease dynamics is investigated in this paper. The infectious population is structured according to time and age of infection. An explicit formula for the basic reproductive number, $R_{0}$ of the model is obtained. We showed that the disease-free equilibrium (DFE) state is locally asymptotically stable if $R_{0}<1$ and unstable if otherwise. This simply means that tuberculosis could be controlled in a population when the basic reproduction number is less than unity. (C) JASEM
\end{abstract}

http://dx.doi.org/10.4314/jasem.v19i4.14

KEYWORDS: Basic reproduction number; Infection-age; Local Stability; Tuberculosis

\section{Introduction}

Tuberculosis otherwise known as tubercle bacillus (TB) is one of the major infectious diseases claiming lives in the world especially in developing countries. It is an infectious disease that spreads through the air and its causative agent amongst the mycobacteria family are mainly the mycobacterium tuberculosis. A susceptible individual needs to inhale only a small number of germs released into the air by an infectious person to be infected. A latent TB infected person does not show any symptoms of the disease and cannot infect others though may live as long as possible without it generating into active TB (WHO, 2007).

The epidemiology of tuberculosis varies substantially around the world (WHO, 2012). It is on record that one third of the world's population are infected with TB. In 2013, 9 million people around the world became sick with TB disease. There were around 1.5 million TB-related deaths worldwide (CDC, 2014). Without treatment, the bacteria will continue to multiply and destroy the body's tissue. Some people develop active TB disease soon after infection (within the first two years), while others develop active TB disease later if their immune system becomes weak, such as individuals with diabetes or those who are infected with HIV (CDC, 2014).

Despite some successes associated with the use of Bacilli Calmette-Guerin (BCG) vaccines and some TB treatment therapies, the disease has continued to increase and has led to the global warning that new control strategies be developed for the total eradication of the disease (Blower et al., 1995). In spite of the wide usage of BCG vaccine, its effectiveness in preventing TB is still controversial (Castillo-Chavez and Feng, 1998). In order to effectively control the spread of TB, a number of authors have proposed mathematical models to study the dynamics, control and prevention of the disease using ordinary differential equations (Guo, 2005).

One of the major reasons that contribute to the rise in TB among developing countries is the inability of the susceptible individuals to go for regular check-ups. Early detection of an ailment helps treatment to be faster while late detection slows down the rate of recovery. In disease modelling, we refer to the various stages of infection as the infection age. Age structure is an important characteristic in the modelling of infectious diseases (Brauer et al., 2013). It provides an effective means of understanding various biological phenomena for large populations (Mahaffy et al., 1998). The incorporation of agestructure leads to a model that includes partial differential equations (PDEs), which makes it more difficult for theoretical analysis (Webb, 1985). Incorporating individual's infection age in epidemic models have been researched upon extensively in a number of articles. The results in those articles show that the age-structure plays an important role in the transmission dynamics of infectious diseases (Huang et al., 2012 and Iannelli et al., 2005).

The organization of this paper is as follows: In the next section, we present the formulation of our model equations and obtain an explicit formula for the reproductive number of infection, which determines the local stability of the disease-free equilibrium 
(DFE) state. We also establish the existence of both the disease-free and endemic equilibrium points.

\section{MATERIALS AND METHODS}

We begin our model formulation by first introducing the model by Ashezua (2011) on A mathematical model to study the effects of treatment and vaccination on the dynamics of tuberculosis in a varying population. The model equations are as follows:

$$
\begin{aligned}
& \frac{d V}{d t}=f v S-\mu N \\
& \frac{d S}{d t}=b N-f v S-\alpha I_{A} S+T_{A} I_{A}+T_{L} I_{L}-\mu S \\
& \frac{d I_{L}}{d t}=(1-P) \alpha I_{A} S-\beta_{A} I_{L}-T_{L} I_{L}-\mu I_{L} \\
& \frac{d I_{A}}{d t}=P \alpha I_{A} S+\beta_{A} I_{L}-T_{A} I_{A}-(\mu+\varepsilon) I_{L} \\
& N=S+V+I_{L}+I_{A}
\end{aligned}
$$

where $N$ represents the total population size at time $t, f$ is the efficacy of the vaccines, $v$ is the vaccination rate, $P$ is the proportion of infection instantaneously degenerating into active TB, $T_{L}$ is the latent TB therapy rate, $T_{A}$ is the active TB treatment cure rate, $\alpha$ is the TB instantaneous incidence rate, $b$ is the population birth rate, $\mu$ represents the population death rate, $\varepsilon$ is the TB-induced death rate and $\beta_{A}$ is the breakdown rate from latent to active TB disease. The existence and stability analysis of the DFE state was established and was found to be locally asymptotically stable, under prescribed conditions on the given model parameters. Furthermore, results from the numerical experiments suggest that, treatment alone could be an effective method of control/ eradication of TB. It was concluded that since many people find it difficult to be confined for long periods of treatment, vaccination of the susceptible should be adopted as an alternative control strategy. In the model, it was assumed that once an individual who has contracted TB is treated, such an individual moves to the susceptible class. So no recovered compartment was considered in their work. More importantly, the model did not consider the fact that the recovering rate of an individual is dependent on the age of infection of the disease. Also, the death rate due to active TB was represented as a parameter. In view of the above, we have formulated a new mathematical model for tuberculosis disease dynamics incorporating infection age structure, recovering rate due to drug administration and death rate due to active TB in the infectious class. The recovering rate due to drug administration and death rate due to active TB are not represented as a parameter in our model. They are all represented in exponential forms. Our model equations are as shown below:

$$
\begin{aligned}
& \frac{d S(t)}{d t}=(1-v) \beta-\alpha S(t) I(t)-\mu S(t) \\
& \quad+\omega V(t)+\int_{0}^{t} \psi(\tau) i(t, \tau) d \tau \\
& \frac{d V(t)}{d t}=v \beta-(\mu+\omega) V(t) \\
& \frac{d L(t)}{d t}=(1-\theta) \alpha S(t) I(t)-(\gamma+\mu) L(t) \\
& \frac{\partial i(t, \tau)}{\partial t}+\frac{\partial i(t, \tau)}{\partial \tau}+(\mu+\sigma(\tau)+\psi(\tau)) i(t, \tau)=0 \\
& i(t, 0)=B(t)=\theta \alpha S(t) I(t)+\gamma L(t) \\
& i(0, \tau)=\varphi(\tau) \\
& \text { where } \\
& I(t)=\int_{0}^{T} i(t, \tau) d \tau \\
& \sigma(\tau)=\delta_{1} e^{-k_{1}(T-\tau)} \\
& \psi(\tau)=\delta_{2} e^{-k_{2}(\tau-T)} \\
& S(0)=S_{0}, V(0)=V_{0}, L(0)=L_{0}, I(0)=I_{0}
\end{aligned}
$$

The variables and parameter of our model equations are presented below:

$V(t)$ : Vaccinated individuals at time, $t$.

$S(t)$ : Susceptible individuals at time, $t$.

$L(t)$ : Latently infected individuals at time, $t$.

$I(t)$ : Actively infected individuals at time, $t$.

$\tau: \quad$ Age of infection.

$T: \quad$ Maximum time of infection.

$\psi(\tau)$ : Recovery rate of the infected class due to drug administration.

$\sigma(\tau)$ : Death rate due to active TB infection.

$\alpha: \quad$ Rate of contracting TB infection.

$\beta$ : Total number of new births joining the total population.

$\mu$ : Natural death rate for the population.

$\theta$ : Proportion of the susceptible moving into the infectious class. And thus,

$(1-\theta)$ : Proportion of the susceptible moving into the latent class.

$\gamma$ : Breakdown rate of an individual from the latent class to the infectious class.

$v$ : Effective vaccination rate.

$k_{1}$ : Effectiveness of the drug therapy.

$k_{2}$ : Ineffectiveness of the drug therapy.

$\delta_{1}$ : Maximum death rate due to infection. 
$\delta_{2}$ : Maximum recovery rate due to drug administration.

$\omega$ : The rate at which the $\mathrm{BCG}$ vaccine wanes

\section{RESULTS AND DISCUSSION}

We re-write equations (6)-(12) as:

$$
\begin{aligned}
& \frac{d S(t)}{d t}=\Lambda-\alpha S(t) \int_{0}^{T} i(t, \tau) d \tau- \\
& \mu S(t)+\omega V(t)+\int_{0}^{t} \psi(\tau) i(t, \tau) d \tau \\
& \frac{d V(t)}{d t}=a-c V(t) \\
& \frac{d L(t)}{d t}=e S(t) \int_{0}^{T} i(t, \tau) d \tau-d L(t) \\
& \frac{\partial i(t, \tau)}{\partial t}+\frac{\partial i(t, \tau)}{\partial \tau}+(\mu+\sigma(\tau)+\psi(\tau)) i(t, \tau)=0(19) \\
& i(t, 0)=B(t)=b S(t) \int_{0}^{T} i(t, \tau) d \tau+\gamma L(t)
\end{aligned}
$$

where

$\Lambda=(1-v) \beta ; \quad a=v \beta ; \quad b=\theta \alpha ; \quad c=(\mu+\omega) ;$ $d=(\gamma+\mu)$ and $e=(1-\theta) \alpha$.

Solving our system of equations (16)-(20) simultaneously, at the disease-free equilibrium state, we arrived at the following points:

$(S, V, L, i(\tau))=\left(\left(\frac{\Lambda}{\mu}+\frac{v \beta \omega}{\mu(\mu+\omega)}\right), \frac{v \beta}{(\mu+\omega)}, 0,0\right)(21)$

Let, $\left(S^{*}, V^{*}, L^{*}, i^{*}(\tau)\right)$ represents any arbitrary endemic equilibrium of the model (16)-(20). This equilibrium satisfies the following equations

$$
\begin{aligned}
& \Lambda-\mu S^{*}+\omega V^{*}-\alpha S^{*} \int_{0}^{T} i^{*}(\tau) d \tau \\
& +\int_{0}^{t} \psi(\tau) i^{*}(\tau) d \tau=0 \\
& a-c V^{*}=0 \\
& e-S^{*} \int_{0}^{T} i^{*}(\tau) d \tau-d L^{*}=0 \\
& \frac{d i^{*}(\tau)}{d \tau}+(\mu+\sigma(\tau)+\psi(\tau)) i^{*}(\tau)=0 \\
& i^{*}(0)=b S^{*} \int_{0}^{T} i^{*}(\tau) d \tau+\gamma L^{*}
\end{aligned}
$$

From (23), we have

$$
V^{*}=\frac{a}{c}=\frac{v \beta}{(\mu+\omega)}
$$

From (24) and (25) we obtained

$$
L^{*}=\frac{\alpha(1-\theta) S^{*}}{(\gamma+\mu)} \int_{0}^{*} i^{*}(0) \pi(\tau) d \tau
$$

and

$$
i^{*}(\tau)=i^{*}(0) \pi(\tau)
$$

where

$$
\pi(\tau)=e^{-\mu \tau} e^{-\int_{0}^{\tau}(\sigma(s)+\psi(s)) d s}
$$

Substituting (27) and (29) into (22) yields

$$
\begin{aligned}
\alpha\left(\frac{\Lambda}{\mu}+\frac{v \beta \omega}{\mu(\mu+\omega)}\right) \int_{0}^{T} i^{*}(0) \pi(\tau) d \tau \\
\\
-\int_{0}^{t} \psi(\tau) i^{*}(0) \pi(\tau) d \tau=0
\end{aligned}
$$

Substituting (28) and (29) into (26) gives

$$
S^{*}=\frac{1}{\left(\alpha \theta \int_{0}^{T} \pi(\tau) d \tau+\frac{\alpha \gamma(1-\theta)}{(\gamma+\mu)} \int_{0}^{T} \pi(\tau) d \tau\right)}
$$

So the endemic equilibrium state $\left(S^{*}, V^{*}, L^{*}, i^{*}(\tau)\right)$ is thus given by (27), (28), (29) and (32).

Let

$$
\begin{aligned}
R_{0}=\alpha & \left(\frac{\Lambda}{\mu}+\frac{v \beta \omega}{\mu(\mu+\omega)}\right) \int_{0}^{T} i^{*}(0) \pi(\tau) d \tau \\
& +\frac{\alpha(1-\theta)}{(\gamma+\mu)}\left(\frac{\Lambda}{\mu}+\frac{v \beta \omega}{\mu(\mu+\omega)}\right) \int_{0}^{T} i^{*}(0) \pi(\tau) d \tau
\end{aligned}
$$

According to Diekmann et al., (1990) $R_{0}$ in (33) can be regarded as the basic reproduction number of our TB model and this is explained as follows. Since the total infectivity at time $t$ is the sum of the infectivities of each infected compartment, we define

$$
R_{0}=R_{i}+R_{L}
$$

where

$$
R_{i}=\alpha S_{0} \int_{0}^{T} i^{*}(0) \pi(\tau) d \tau
$$

is the number of secondary cases generated by individuals in the actively infected class and $S_{0}=\left(\frac{\Lambda}{\mu}+\frac{v \beta \omega}{\mu(\mu+\omega)}\right)$ is the number of susceptible individuals in the absence of TB. The term:

$$
\pi(\tau)=e^{-\int_{0}^{\tau}(\mu+\sigma(s)+\psi(s)) d s}
$$

is the survival probability as a function of age $\tau$ in the actively infected class.

For

$$
R_{L}=\frac{\alpha(1-\theta) S^{*}}{(\gamma+\mu)} \int_{0}^{T} i^{*}(0) \pi(\tau) d \tau
$$

$\alpha(1-\theta)$ is the proportion of the susceptible individuals acquiring latent TB infection. 
We consider the local stability of the DFE state $(S, V, L, i(\tau))=\left(\left(\frac{\Lambda}{\mu}+\frac{v \beta \omega}{\mu(\mu+\omega)}\right), \frac{v \beta}{(\mu+\omega)}, 0,0\right)=E_{0}$

Let,

$$
\begin{aligned}
& S(t)=\left(\frac{\Lambda}{\mu}+\frac{v \beta \omega}{\mu(\mu+\omega)}\right)+x(t) \\
& V(t)=\frac{v \beta}{(\mu+\omega)}+y(t) \\
& L(t)=q(t) \\
& i(t, \tau)=z(t, \tau)
\end{aligned}
$$

Linearizing equations (16)-(20) about $E_{0}$, we obtain the following equations

$$
\begin{aligned}
\frac{d x(t)}{d t}= & -\mu x(t)+\omega y(t) \\
& -\alpha\left(\frac{\Lambda}{\mu}+\frac{v \beta \omega}{\mu(\mu+\omega)}\right) \int_{0}^{T} z(t, \tau) d \tau \\
& +\int_{0}^{t} \psi(\tau) z(t, \tau) d \tau \\
\frac{d y(t)}{d t}= & -(\mu+\omega) y(t)+v \beta-\frac{\mu v \beta}{(\mu+\omega)}-\frac{v \beta \omega}{(\mu+\omega)} \\
\frac{d q(t)}{d t}= & \alpha(1-\theta)\left(\frac{\Lambda}{\mu}+\frac{v \beta \omega}{\mu(\mu+\omega)}\right) \int_{0}^{T} z(t, \tau) d \tau \\
& -(\gamma+\mu) q(t) \\
\frac{\partial z(t, \tau)}{\partial t} & +\frac{\partial z(t, \tau)}{\partial \tau}+(\mu+\sigma(\tau)+\psi(\tau)) z(t, \tau)=0 \\
z(t, 0)= & \alpha \theta\left(\frac{\Lambda}{\mu}+\frac{v \beta \omega}{\mu(\mu+\omega)}\right) \int_{0}^{T} z(t, \tau) d \tau-\gamma q(t)
\end{aligned}
$$

To analyse the asymptotic behaviour of $E_{0}$, we look for solutions of the form $x(t)=\bar{x} e^{\lambda t}$,

$y(t)=\bar{y} e^{\lambda t}, q(t)=\bar{q} e^{\lambda t}$ and $z(t, \tau)=\bar{z}(\tau) e^{\lambda t}$. Thus, we can consider the following eigen-value problem.

$$
\begin{aligned}
& (\mu+\lambda) \bar{x}=\omega \bar{y}-\alpha\left(\frac{\Lambda}{\mu}+\frac{v \beta \omega}{\mu(\mu+\omega)}\right)^{T} \int_{0}^{-} \bar{z}(\tau) d \tau \\
& +\int_{0}^{t} \psi(\tau) \bar{z}(\tau) d \tau \\
& (\mu+\omega+\lambda) \bar{y}=v \beta-\frac{\mu v \beta}{(\mu+\omega)}-\frac{v \beta \omega}{(\mu+\omega)} \\
& (\gamma+\mu+\lambda) \bar{q}=\alpha(1-\theta)\left(\frac{\Lambda}{\mu}+\frac{v \beta \omega}{\mu(\mu+\omega)}\right) \int_{0}^{T} \bar{z}(\tau) d \tau( \\
& \frac{d \bar{z}(\tau)}{d \tau}=-(\lambda+\mu+\sigma(\tau)+\psi(\tau)) \bar{z}(\tau) \\
& \bar{z}(0)=\alpha \theta\left(\frac{\Lambda}{\mu}+\frac{v \beta \omega}{\mu(\mu+\omega)}\right) \int_{0}^{T} \bar{z}(\tau) d \tau+\gamma \bar{q}
\end{aligned}
$$

Solving (45) for $\bar{z}(\tau)$ we obtain

$\bar{z}(\tau)=\bar{z}(0) e^{-(\lambda+\mu+\sigma(\tau)+\psi(\tau)) \tau}$

Substituting (47) into (46) gives

$\bar{q}=\frac{\bar{z}(0) e S_{0}}{(\gamma+\mu+\lambda)} \int_{0}^{T} e^{-\lambda \tau} \pi(\tau) d \tau$

Substituting (48) into (46), gives $1=b S_{0} \int_{0}^{T} e^{-\lambda \tau} \pi(\tau) d \tau+\frac{\gamma e S_{0}}{(\gamma+\mu+\lambda)} \int_{0}^{T} e^{-\lambda \tau} \pi(\tau) d \tau$

Define a function $G(\lambda)$ to be the right hand side in (49). Obviously, $G(\lambda)$ is continuously differentiable function with $\lim _{\lambda \rightarrow \infty} G(\lambda)=0$. By direct computation, it can be shown that $G^{\prime}(\lambda)<0$, and therefore, $G(\lambda)$ is a decreasing function. Hence, any real solution of equation (49) is negative if $G(0)<1$, and positive if $G(0)>1$.Thus, if $G(0)>1$, the DFE state is unstable.

Next, we show that equation (49) has no complex solution with non-negative real part if $G(0)<1$. In fact, we set

$$
\begin{aligned}
& H(\tau)=b S_{0} \pi(\tau) \\
& F(\tau)=\gamma e S_{0} \pi(\tau)
\end{aligned}
$$

Thus, we have

$G(\lambda)=\int_{0}^{T} e^{-\lambda \tau} H(\tau) d \tau+\frac{1}{(\gamma+\mu+\lambda)} \int_{0}^{T} e^{-\lambda \tau} F(\tau) d \tau$

Suppose $G(0)<0$. Assume that $\lambda=a_{1}+b_{1} i$ is a complex solution of equation (52) with $a_{1} \geq 0$. Then,

$$
\begin{aligned}
& |G(\lambda)|=\left|\int_{0}^{T} e^{-\lambda \tau} H(\tau) d \tau+\frac{1}{(\gamma+\mu+\lambda)} \int_{0}^{T} e^{-\lambda \tau} F(\tau) d \tau\right| \\
& \leq\left|\int_{0}^{T} e^{-\left(a_{1}+i b_{1}\right) \tau} H(\tau) d \tau\right|+\frac{1}{\left|\gamma+\mu+a_{1}+i b_{1}\right|}\left|\int_{0}^{T} e^{-\left(a_{1}+i b_{1}\right) \tau} F(\tau) d \tau\right|
\end{aligned}
$$$$
=\int_{0}^{T}\left|e^{-\left(a_{1}+i b_{1}\right) \tau}\right| H(\tau) d \tau+\frac{1}{\sqrt{\left(a_{1}+\gamma+\mu\right)^{2}+b_{1}^{2}}} \int_{0}^{T}\left|e^{-\left(a_{1}+i b_{1}\right) \tau}\right| F(\tau) d \tau
$$$$
\leq \int_{0}^{T} e^{-a_{1} \tau} H(\tau) d \tau+\frac{1}{a_{1}+\gamma+\mu} \int_{0}^{T} e^{-a_{1} \tau} F(\tau) d \tau
$$$$
=\left|G\left(a_{1}\right)\right| \leq G(0)<1
$$

It follows from equation (53) that equation (52) has solutions $\lambda=a_{1}+i b_{1}$ only if $a_{1}<0$. Thus, every solution of (52) must have a negative real part. Observe that $R_{0}=G(0)$. Therefore, the DFE state $E_{0}$ is locally asymptotically stable if $G(0)<1$.

Thus, we have proved the following theorem. 
Theorem: The disease-free equilibrium state $E_{0}$ is locally asymptotically stable if $R_{0}<1$ and unstable if $R_{0}>1$.

In this paper, we first formulate a mathematical model for tuberculosis disease dynamics incorporating infection age-structure using ordinary differential equation, integro-differential equation and partial differential equation. We obtain an explicit formula for the reproduction number of our model. We equally obtained the existence of both the disease-free and endemic equilibrium points. Finally we established the fact that the disease-free equilibrium state is locally asymptotically stable for $R_{0}<1$. This simply means that TB could be controlled when the basic reproduction number is below one.

Acknowledgement: The first author wishes to thank the University of Agriculture, Makurdi for granting him study fellowship during which this research was carried out.

\section{REFERENCES}

Ashezua, T.T. (2011). A mathematical model to study the effects of treatment and vaccination on the dynamics of tuberculosis in a varying population. An M.Sc. thesis, University of Agriculture, Makurdi, Nigeria. (Unpublished).

Blower, S.M., McLean, A.R., Porco, T.C., Small, P.M., Hope, P.C., Sanchez, M.A. and Moss, A.R. (1995). The intrinsic transmission dynamics of tuberculosis epidemics, Nat. Med. Vol 1, No. 8, pp. 815-821.

Brauer, F., Shuai, Z. and Van Den Driessche, P. (2013). Dynamics of an age-of- infection Cholera model, Mathematical Bioscience and Engineering, Vol. 10, No. 5 \& 6, pp. 1335-1349.

Castillo-Chavez, C. and Feng, Z. (1998). Global stability of an age-structured model for TB and its applications to optimal vaccination strategies. Mathematics Biosciences, Vol. 151, pp. 135-154.

Centre for Disease Control and Prevention (2014). Tuberculosis: An overview. Factsheet. Available online at: web page: www.cdc.gov

Diekmann, O, Heesterbeek, J.A.P. and Metz, J.A.J. (1990). On the definition and the computation of the basic reproduction ratio in models for infectious diseases in heterogeneous populations, J. Math. Biol., Vol. 28, pp. 365-382.

Guo, H. (2005). Global dynamics of a mathematical model of tuberculosis, Canadian Applied Mathematics Quarterly, Vol. 13, No. 4, Winter.

Huang, G., Liu, X. and Takeuchi, Y. (2012). Lyapunov functions and global stability for agestructured HIV infection Model, SIAM J. Appl. Math., Vol. 72, pp.25-38.

Iannelli, M., Martcheva, M. and Li, X.Z. (2005). Strain replacement in an epidemic modelwith superinfection and perfect vaccination, Math. Biosci., Vol.195, pp.845-862.

Mahaffy, J.M., Belair, J. and Mackey, M. (1998). Hematopoietic model with moving boundary condition and state dependent delay: Applications in erythropoiesis. J. theor. Biol., Vol. 135, pp. 135-146.

Webb, G. (1985). Theory of nonlinear age-dependent population dynamics, Marcel Dekker, New York.

World Health Organization (2007). WHO fact sheet. Technical report 104, stop TBdepartment, Geneva, Switzerland. Web pagewww.who.org

World Health Organization (2012). WHO fact sheet. Technical report 104, stop TBdepartment, Geneva, Switzerland. Web page: www.who.org 